\title{
Comparative Studies on Leaf Yield, Quality and Economy of Three Cultivars of Betelvine (Piper betle L.) as Influenced by Integrated Nitrogen Management
}

\author{
Aloke Bar ${ }^{1 *}$, Anupam Pariari ${ }^{1,}$ Pooja Biswas $^{2}$, Milan Karmakar ${ }^{1}$ and Gobinda Saha ${ }^{1}$ \\ ${ }^{1}$ Department of Plantation, Spices, Medicinal and Aromatic Crops, ${ }^{2}$ Department of Vegetable \\ Science, Bidhan Chandra Krishi Viswavidyalaya, Mohanpur, Nadia, West Bengal, India \\ *Corresponding author
}

\section{A B S T R A C T}

\section{Keywords}

Simurali Deshi, Simurali Bhabna, Halisahar Sanchi, Mustard oil cake, Cow dung manure, Poultry manure, Pond silt, Fresh and dry weight,

Chlorophyll content, Essential oil content, Ascorbic acid content, B Carotene content

\section{Article Info}

Accepted:

22 October 2020

Available Online:

10 November 2020
Piper betle L., (family- Piperaceae) commonly known as the betel vine is an important medicinal and recreational plant in Southeast Asia. Betel vine is a dioecious, evergreen, perennial creeper with glossy heart shaped leaves. The present study was executed to identify the suitable integrated nitrogen management on leaf yield, quality and economy of betel vine (Piper betle L.) cultivars in new alluvial zone of West Bengal. The field experiment was conducted at Horticulture Research Station, Mondouri, Bidhan Chandra Krishi Viswavidyalaya, Nadia, West Bengal during the 2017-2018. The experiment was laid out in Rnadomized Block Design, replicated thrice with eight treatments of integrated nitrogen management. It was noted that treatments in combination with inorganic and organic sources of nitrogen produced better results than the treatments with sole application of organic source of nitrogen. The application of nitrogen from mustard oil cake and urea in 1:1 ratio $\left(\mathrm{T}_{1}\right)$ produced maximum leaf yield/ vine (86.45 leaves/ vine/ year), projected leaf yield (63.22 lakhs/ ha/ year), chlorophyll content of the leaves (52.30 SPAD value), Ascorbic acid content of leaves $(3.07 \mathrm{mg} / 100 \mathrm{~g})$, essential oil content $(65.50 \mathrm{mg} / 100 \mathrm{~g})$ and $B$ Carotene content of leaves $(7.28 \mathrm{mg} / 100 \mathrm{~g})$ in Simurali Deshi $\left(\mathrm{V}_{1}\right)$. Maximum fresh weight $(261.25 \mathrm{~g})$ and dry weight (42.01 g) of 100 leaves were found in Halisahar Sanchi $\left(\mathrm{V}_{3}\right)$. Among all the sources of nitrogen Mustard oil cake 50\% + Urea 50\% $\left(\mathrm{T}_{1}\right)$ showed highest benefit and cost ratio in all the three cultivars.

\section{Introduction}

Betel vine (Piper betle Linn) commonly known as Paan is a perennial dioecious evergreen creeper, grown in shady condition with high humidity (Das and Mallick, $2010)^{[7]}$. It is a member of Piperaceae family.
It is originated from Malaysia but is distributed extensively in South and Southwest China. This crop is usually cultivated in India, Sri Lanka, Malaysia, Thailand, Taiwan and other Southeast Asian countries. This plant is economically, medicinally and traditionally important in the 
whole world (Punuri et al., 2012) ${ }^{[26]}$. It is one of the important commercial crop grown mostly by small and marginal farmers in the states of Assam, West Bengal, Bihar, Andhra Pradesh, Karnataka, Tamil Nadu, Maharashtra, Madhya Pradesh, Kerala, Uttar Pradesh and Orissa with an estimated area of 53,539 ha (Ray, 2008) ${ }^{[30]}$. The cultivation economy depends both in terms of benefit-tocost ratio and net income. For the last 7 years an average productivity of 19.41 lakh leaves/ha has been observed (Kaleeswari and Sridhar, 2013) ${ }^{[17]}$. India has exported 13195.43 MT of Betel Leaves to the world for the worth of Rs. 46.75 crores/ 6.74 USD Millions in 2018-19 (APEDA agriXchange) [1]. It is a perennial source of employment (Prasad et al., 2003) ${ }^{[25]}$. The betelvine is called as 'green gold of india' as about 20 million people derive their livelihood directly or indirectly from production, processing, handling, transportation and marketing of betel leaves in India (Janeet al., 2014) ${ }^{[15]}$. Betel vine ( $P$. betle) is called by different names in India i.e. Pan in Hindi, Tambula in Sanskrit, Villayadela in Kannada, Vettilakkoti in Malayalam, Vettilai in Tamil, Tamalapaku in Telugu, Videch-pan in Marathi, Nagarbel in Gujrati, Pan in Bangala also called Tanbol in Arabic and Burg-e-Tanbol in Persian. (Thomas et al., 2013) ${ }^{[37]}$. The betel leaves are mainly used as mouth freshener and is also well known for curing many communicable and non-communicable diseases like cold, cough, bronchial asthma, rheumatism, stomachalgia and used to treat other diseases like bad breath, boils and abscesses, conjunctivitis, constipation, swelling of gums, cuts and injuries (Gundala et al., 2014) ${ }^{[12]}$. It is also found that betel has significant anti proliferative activity in vitro and in vivo prostate cancer models (Paranjpeet al., 2013) [23]. Studies have evidenced that some potential phytochemicals of the betel vine leaf extract such as hydroxychavicol (Chang et al., 2002; Jeng et al., 2004) ${ }^{[5,16]}$, ursolic acid
(Kassi et al., 2007; Yamai et al., 2009; Yu et al., 2010; Shao et al., 2011) ${ }^{[18,40,41,35]}$, chlorogenic acid (Rakshit et al., 2010) ${ }^{[28]}$, and eugenol (Ghosh et al., 2005; Pisano et al., 2007; Manikandan et al., 2010; Jaganathan et al., 2011) ${ }^{[9,24,22,14]}$ can induce apoptosis in tumour cells confirming the chemopreventive effect. The crop is cultivated in conservatory structure called as 'boroj.'It provides shade and high humid environment to the crop needed for a good harvest (Khatua, 2013) ${ }^{[19]}$. Lack of soil moisture (drought), insufficient water supply, occurrence of natural calamities, disease and pest attack, nonavailability of skilled labour, high labour cost, lack of storage facilities, transportation facilities, large number of intermediaries, lack of export promotional activities etc. are some constraints of betel vine cultivation (Sahoo et al., 2017) ${ }^{[13]}$. The betel leaf can be segregated into various varieties based on the leaf size, colour, taste, and fragrance. Some of the commonly cultivated varieties of betel vine in India are the Mysore, Banarasi, Salem, Magadhi, Kauri, Calcutta, Bagerhati, and Ghanagete (Satyavati et al., 1987; Warrier et al., 1995; Rai et al., 2011) ${ }^{[33,38,27]}$. The potential growth and developmental rates for a particular genotype may be decreased by stress factors (Shukla et al., 2013) ${ }^{[36]}$.Light is one of the most limiting factors for growth and biomass of the plant (Yuan-long et al., $2012)^{[42]}$. Since the green leaves constitute the economically relevant part of the plant, the nitrogen requirement of the crop is quite high (Saikia et al., 1995) $^{[32]}$. In India most of the betel vine growers apply large amounts of organic manures mainly in the form of mustard oil cake. Use of integrated nitrogen management with balanced organic and inorganic nitrogen sources can provide cost effective and environment friendly production tool. This present study assesses the effect of different integrated nitrogen management treatments in betel vine (Piper betle L.) cultivars in new alluvial zone of West Bengal. 


\section{Materials and Methods}

The experiment was laid out in Rnadomized Block Design with three replications. Three cultivars viz., Simurali Deshi $\left(\mathrm{V}_{1}\right)$, Simurali Bhabna $\left(\mathrm{V}_{2}\right)$ and Halisahar Sanchi $\left(\mathrm{V}_{3}\right)$ were used. The eight treatments of integrated nitrogen management viz., $\mathrm{T}_{1}=$ Mustard oil cake $(50 \%)+$ Urea $(50 \%), \mathrm{T}_{2}=$ Cow dung manure $(50 \%)+$ Urea $(50 \%), \mathrm{T}_{3}=$ Poultry manure $(50 \%)+$ Urea $(50 \%), \mathrm{T}_{4}=$ Pond silt $(50 \%)+$ Urea $(50 \%), \mathrm{T}_{5}=$ Mustard oil cake $(100 \%), \mathrm{T}_{6}=$ Cow dung manure $(100 \%), \mathrm{T}_{7}=$ Poultry manure $(100 \%)$ and $\mathrm{T}_{8}=$ Pond silt $(100 \%)$. Experiment was conducted at Horticulture Research Station, Mondouri, Bidhan Chandra Krishi Viswavidyalaya, Nadia, West Bengal. The experiment site is located at $23^{\circ} 50^{\prime} \mathrm{N}$ latitude and $80^{\circ} 02^{\prime} \mathrm{E}$ longitude with an altitude of 9.75 meters above mean sea level (MSL). The soil of the experimental site is sandy loam in texture having good drainage and water holding capacity and slightly acidic in nature with moderate soil fertility status. Recommended dose of fertilizers for betel vine is 200:100:100 kg NPK/ha, N applied in the form of urea, mustard oil cake, cow dung manure, poultry manure and pond silt, $\mathrm{P}_{2} \mathrm{O}_{5}$ applied in the form of single super phosphate and $\mathrm{K}_{2} \mathrm{O}$ applied in the form of MOP. In all the nitrogen sources $\mathrm{P}_{2} 0_{5} @ 100 \mathrm{~kg} / \mathrm{ha}$ and $\mathrm{K}_{2} \mathrm{O} @ 100 \mathrm{~kg} / \mathrm{ha}$ were applied from inorganic sources during April considering the amount present in the respective organic manures to be applied. Then different combinations of nitrogenous fertilizer were applied in different nitrogen sources in three split doses during April, July and September. Leaf production was recorded in total number of leaves/ vine/ year. Data was collected at monthly interval and then added to get total number of leaves produced by a vine in a year. Projected leaf yield was recorded with the help of leaf production by a vine in a year multiplied by total number of plants covering one hectare of land (number of leaves/ vine/ year $\mathrm{X}$ total number of vines/ ha). It was expressed in lakh/ ha. Fresh weight of leaves was taken from freshly harvested 100 leaves without petiole. For taking dry weight of leaves, the same leaves were dried in a drier at $450 \mathrm{C}$ for $48 \mathrm{~h}$ and weighed. For essential content, $100 \mathrm{~g}$ fresh betel leaves from each cultivar were taken for extraction and estimation of essential oil content. The essential oil was extracted using Clevenger's method $(1928)^{[6]}$. The volume of the essential oil was collected in $10 \mathrm{ml}$ conical flask and calculated on weight basis. Chlorophyll content of the randomly selected nine leaves per plot was measured after harvesting of leaves at an interval of six months by using portable leaf chlorophyll meter (SPAD 502, Minolta) and following the method of Arnon $(1949)^{[2]} \cdot \beta$-carotene in leaf was estimated by the method of Broadman \& Anderson $(1967)^{[4]}$. Ascorbic acid was estimated by using 2, 6 dichlorophenol indophenol dye titration method (Ranganna, 2000) ${ }^{[29]}$. The present experiment is designed in factorial RBD (Randomized Block Design) with three replications, where nitrogen sources and cultivars were considered as two factors. The data obtained in this experiment were subjected to statistical analysis by appropriate analysis of variance method (Gomez and Gomez, 1984) ${ }^{[10]}$ and significance of different sources of variations were tested by Fisher and Snedecor's ' $F$ ' test at 0.05 probability level.

\section{Results and Discussion}

\section{Yield characters}

\section{Leaf yield/ vine/ year}

The economic part of betel vine is leaf so importance is always given on more leaf production/ vine/ year. Data presented in Table 1 clearly reveals that nitrogen sources, 
cultivars and their interaction had a significant influence on leaf yield/ vine/ year. It was noted from the experimental findings that among the sources of nitrogen Mustard oil cake $50 \%+$ Urea $50 \%\left(\mathrm{~T}_{1}\right)$ resulted highest leaf yield/ vine in all of the three cultivars with the number of leaves of 86.45 leaves/ vine/ year in Simurali Deshi $\left(\mathrm{V}_{1}\right)$, 81.01 leaves/ vine/ year in Simurali Bhabna $\left(\mathrm{V}_{2}\right)$ and 81.93 leaves/ vine/ year in Halisahar Sanchi $\left(\mathrm{V}_{3}\right)$ followed by Cowdung manure $50 \%$ + Urea $50 \%\left(\mathrm{~T}_{2}\right)$. The best performance was shown with the application of mustard oil cake $(50 \%)$ and urea $(50 \%)$ as the nitrogen source maximum number of leaves (83.13 leaves/ vine) were produced/ vine and when total nitrogen was only from the organic sources $(100 \%)$ the vine showed lowest number of leaf (42.81 leaves/ vine). Data presented in Table 1 reveals that among the cultivars highest overall number of leaves/ vine/ year was obtained 59.75 leaves/ vine /year in Simurali Deshi $\left(\mathrm{V}_{1}\right)$ followed by 57.20 leaves/ vine /year in Halisahar Sanchi $\left(\mathrm{V}_{3}\right)$ and 55.37 leaves/ vine /year in Simurali Bhabna $\left(\mathrm{V}_{2}\right)$. Similar results were found by Maiti et al, (1995) ${ }^{[21]}$, Saikia et al, $(1995)^{[32]}$, Dey et al., $(2003)^{[8]}$, Das et al., $(2010)^{[7]}$, and Imam et al., $(2012)^{[13]}$.

\section{Projected leaf yield/ ha/ year}

The data presented in Table- 2 indicates that nitrogen sources and cultivars registered their significant effect on projected leaf yield/ hectare/ year. Among the sources of nitrogen Mustard oil cake $50 \%$ + Urea $50 \%\left(\mathrm{~T}_{1}\right)$ resulted highest leaf yield/ha/year resulting 63.22 lakhs leaves/ year/ ha in Simurali Deshi $\left(\mathrm{V}_{1}\right), 59.92$ lakhs leaves/ year/ ha in Halisahar Sanchi $\left(\mathrm{V}_{3}\right)$ and 59.24 lakhs leaves/ year/ ha in Simurali Bhabna $\left(\mathrm{V}_{2}\right)$. The best performance was shown with the application of mustard oil cake (50\%) and urea (50\%). Among the cultivars, Simurali Deshi $\left(\mathrm{V}_{1}\right)$ performed the best (43.70 lakhs leaves/ year/ ha). Dey et al., (2003) ${ }^{[8]}$, revealed that highest leaf yield (74.67 lakhs/ha) was obtained when the required nitrogen was applied from Mustard oil cake 50\% + Urea 50\%). Imam et al., (2012) ${ }^{[13]}$ reported that the highest number of leaf (77.37 lakh /ha) was obtained from mustard oil cake and urea at 50:50 combination.

Maximum leaf yield was recorded when organic and inorganic nitrogen was applied in equal proportion. This might be due to maximum utilization of nitrogen from inorganic and organic sources. Urea is a water soluble fertilizer, and prone to high leach ability in the soil. Therefore, if nitrogen was applied fully through urea, then leaching loss was more and plants could not utilize nitrogen totally. On the other hand, the utilization of nitrogen was found better when it was applied with a combination from both the sources. Although on the basis of content and utilization of nitrogen, organic manures are less efficient than the inorganic fertilizers, combined use of those sources was considered to be superior to use of either organic or inorganic nitrogen alone.

The reason behind those observations may be that urea hastened decomposition of mustard oil cake by providing a part to the microorganism to mobilize nitrogen slowly and progressively from the organic sources. This promoted to attain faster growth rate at early period, which reflected on overall impact of vine and leaf yield. (Dey et al., $2003)^{[8]}$. The superiority of combination, but nitrogen through inorganic source might be added advantage of organic nutrients, which besides supplying all the essential nutrients, also improves physical conditions of soil in respect of granulation, friability, porosity and water holding capacity which enable the crop to utilize nutrients and water more efficiently especially under light textured loamy sand soils (Yadav, 2005) ${ }^{[39]}$. 
Table.1 Effect of different nitrogen sources on leaf yield / vine/ year of three different cultivars of Betel vine

\begin{tabular}{|c|c|c|c|c|}
\hline \multirow{2}{*}{$\begin{array}{c}\text { Nitrogen } \\
\text { source }\end{array}$} & \multicolumn{4}{|c|}{ Cultivar } \\
\hline & $V_{1}$ & $\mathbf{V}_{2}$ & $\mathbf{V}_{\mathbf{3}}$ & Nitrogen source Mean \\
\hline$T_{1}$ & 86.45 & 81.01 & 81.93 & 83.13 \\
\hline $\mathbf{T}_{2}$ & 73.85 & 67.33 & 68.09 & 69.76 \\
\hline $\mathbf{T}_{3}$ & 60.96 & 56.20 & 60.59 & 59.25 \\
\hline $\mathbf{T}_{4}$ & 58.62 & 54.09 & 54.23 & 55.64 \\
\hline $\mathbf{T}_{5}$ & 57.30 & 54.15 & 57.17 & 56.21 \\
\hline $\mathbf{T}_{6}$ & 51.18 & 45.93 & 47.00 & 48.03 \\
\hline $\mathbf{T}_{7}$ & 46.29 & 42.21 & 45.56 & 44.69 \\
\hline$T_{8}$ & 43.40 & 42.00 & 43.02 & 42.81 \\
\hline $\begin{array}{l}\text { Cultivar } \\
\text { Mean }\end{array}$ & 59.75 & 55.37 & 57.90 & \\
\hline \multicolumn{3}{|c|}{ Factors } & C.D. $(p=0.05)$ & $\mathrm{SE}(\mathrm{m}) \pm$ \\
\hline \multicolumn{3}{|c|}{ Nitrogen source (Factor A) } & 1.52 & 0.53 \\
\hline \multicolumn{3}{|c|}{ Cultivar (Factor B) } & 0.93 & 0.33 \\
\hline \multicolumn{3}{|c|}{ Nitrogen source X Cultivar } & 2.64 & 0.92 \\
\hline
\end{tabular}

Note: $\mathrm{T}_{1}=$ Mustard oil cake $(50 \%)+$ Urea $(50 \%), \mathrm{T}_{2}=$ Cow dung manure $(50 \%)+$ Urea $(50 \%), \mathrm{T}_{3}=$ Poultry manure $(50 \%)+$ Urea $(50 \%), \mathrm{T}_{4}=$ Pond silt $(50 \%)+$ Urea $(50 \%), \mathrm{T}_{5}=$ Mustard oil cake $(100 \%), \mathrm{T}_{6}=$ Cow dung manure $(100 \%), \mathrm{T}_{7}=$ Poultry manure $(100 \%), \mathrm{T}_{8}=$ Pond silt $(100 \%)$, Simurali Deshi $\left(\mathrm{V}_{1}\right)$, Simurali Bhabna $\left(\mathrm{V}_{2}\right)$ and Halisahar Sanchi $\left(\mathrm{V}_{3}\right)$

Table.2 Effect of different nitrogen sources on projected leaf yield/ ha/ year of three different cultivars of Betelvine (lakh /ha)

\begin{tabular}{|c|c|c|c|c|}
\hline \multirow[t]{2}{*}{ Nitrogen source } & \multicolumn{3}{|c|}{ Cultivar } & \multirow[b]{2}{*}{ Nitrogen source Mear } \\
\hline & $V_{1}$ & $\mathbf{V}_{2}$ & $\mathbf{V}_{3}$ & \\
\hline$T_{1}$ & 63.22 & 59.24 & 59.92 & 60.79 \\
\hline $\mathbf{T}_{2}$ & 54.00 & 49.24 & 49.79 & 51.01 \\
\hline $\mathbf{T}_{3}$ & 44.58 & 41.10 & 44.31 & 43.33 \\
\hline $\mathbf{T}_{4}$ & 42.86 & 39.55 & 39.66 & 40.69 \\
\hline$T_{5}$ & 41.90 & 39.60 & 41.81 & 41.10 \\
\hline $\mathrm{T}_{6}$ & 37.42 & 33.59 & 34.37 & 35.13 \\
\hline $\mathbf{T}_{7}$ & 33.85 & 30.87 & 33.32 & 32.68 \\
\hline $\mathbf{T}_{8}$ & 31.74 & 30.72 & 31.46 & 31.30 \\
\hline Cultivar Mean & 43.70 & 40.49 & 41.83 & \\
\hline \multicolumn{2}{|c|}{ Factors } & \multicolumn{2}{|c|}{ C.D. $(p=0.05)$} & $\mathrm{SE}(\mathrm{m}) \pm$ \\
\hline \multicolumn{2}{|c|}{ Nitrogen source (Factor A) } & \multicolumn{2}{|c|}{1.11} & 0.39 \\
\hline \multicolumn{2}{|c|}{ Cultivar (Factor B) } & \multicolumn{2}{|c|}{0.68} & 0.24 \\
\hline \multicolumn{2}{|c|}{ Nitrogen source X Cultivar } & & & 0.68 \\
\hline
\end{tabular}

Note: $\mathrm{T}_{1}=$ Mustard oil cake $(50 \%)+$ Urea $(50 \%), \mathrm{T}_{2}=$ Cow dung manure $(50 \%)+$ Urea $(50 \%), \mathrm{T}_{3}=$ Poultry manure $(50 \%)+$ Urea $(50 \%), \mathrm{T}_{4}=$ Pond silt $(50 \%)+$ Urea $(50 \%), \mathrm{T}_{5}=$ Mustard oil cake $(100 \%), \mathrm{T}_{6}=$ Cow dung manure $(100 \%)$, $\mathrm{T}_{7}=$ Poultry manure $(100 \%), \mathrm{T}_{8}=$ Pond silt $(100 \%)$, Simurali Deshi $\left(\mathrm{V}_{1}\right)$, Simurali Bhabna $\left(\mathrm{V}_{2}\right)$ and Halisahar Sanchi $\left(\mathrm{V}_{3}\right)$ 
Table.3 Effect of different nitrogen sources on qualitative characters of three different cultivars of Betelvine

\begin{tabular}{|c|c|c|c|c|c|c|}
\hline Treatments & $\begin{array}{l}\text { Fresh wt of } \\
100 \text { leaves (g) }\end{array}$ & $\begin{array}{l}\text { Dry wt of } \\
100 \text { leaves } \\
\text { (g) }\end{array}$ & $\begin{array}{c}\text { Chlorophyll } \\
\text { Content (SPAD } \\
\text { value) }\end{array}$ & $\begin{array}{c}\text { Essential oil } \\
\text { content } \\
(\mathrm{mg} / \mathbf{1 0 0 g})\end{array}$ & $\begin{array}{l}\text { B Carotene } \\
\text { (mg /100g) }\end{array}$ & $\begin{array}{c}\text { Ascorbic } \\
\text { acid(mg } \\
/ 100 g)\end{array}$ \\
\hline $\mathbf{T}_{1}$ & 253.84 & 41.03 & 50.19 & 59.394 & 7.11 & 2.75 \\
\hline $\mathbf{T}_{2}$ & 250.97 & 40.04 & 48.35 & 58.006 & 6.98 & 2.69 \\
\hline $\mathbf{T}_{3}$ & 244.37 & 38.69 & 47.65 & 56.948 & 6.77 & 2.62 \\
\hline $\mathbf{T}_{4}$ & 235.22 & 36.34 & 45.79 & 56.079 & 6.56 & 2.54 \\
\hline $\mathbf{T}_{5}$ & 239.92 & 37.68 & 45.00 & 55.249 & 6.46 & 2.50 \\
\hline$T_{6}$ & 244.09 & 38.70 & 43.69 & 53.858 & 6.35 & 2.38 \\
\hline $\mathbf{T}_{7}$ & 231.93 & 36.36 & 42.83 & 52.914 & 6.12 & 2.31 \\
\hline $\mathbf{T}_{8}$ & 226.54 & 35.71 & 40.88 & 51.531 & 5.94 & 2.23 \\
\hline CD & 2.76 & 0.49 & 0.39 & 0.57 & 0.07 & 0.01 \\
\hline $\operatorname{S.Em}( \pm)$ & 0.97 & 0.17 & 0.14 & 0.20 & 0.02 & 0.01 \\
\hline$V_{1}$ & 235.42 & 37.52 & 46.72 & 60.68 & 6.75 & 2.82 \\
\hline $\mathbf{V}_{2}$ & 238.96 & 37.78 & 45.62 & 54.18 & 6.49 & 2.54 \\
\hline $\mathbf{V}_{3}$ & 248.20 & 38.90 & 44.29 & 51.63 & 6.38 & 2.16 \\
\hline CD & 1.69 & 0.23 & 0.24 & 0.35 & 0.04 & 0.01 \\
\hline $\operatorname{S.Em}( \pm)$ & 0.59 & 0.11 & 0.08 & 0.12 & 0.01 & 0.01 \\
\hline $\mathrm{T}_{1} \mathrm{~V}_{1}$ & 247.91 & 40.04 & 52.30 & 65.5 & 7.28 & 3.07 \\
\hline $\mathbf{T}_{2} \mathrm{~V}_{1}$ & 244.40 & 39.07 & 49.38 & 63.737 & 7.19 & 3.00 \\
\hline$T_{3} V_{1}$ & 238.15 & 38.00 & 48.85 & 62.577 & 7.06 & 2.93 \\
\hline$T_{4} V_{1}$ & 230.43 & 36.00 & 46.96 & 61.433 & 6.71 & 2.87 \\
\hline $\mathbf{T}_{5} \mathbf{V}_{1}$ & 240.22 & 38.00 & 46.15 & 61.217 & 6.63 & 2.83 \\
\hline$T_{6} V_{1}$ & 234.79 & 38.02 & 44.66 & 58.633 & 6.57 & 2.70 \\
\hline $\mathbf{T}_{7} \mathbf{V}_{1}$ & 226.86 & 36.01 & 43.69 & 57.493 & 6.39 & 2.63 \\
\hline$T_{8} V_{1}$ & 220.60 & 35.01 & 41.76 & 54.857 & 6.15 & 2.54 \\
\hline $\mathbf{T}_{1} \mathbf{V}_{2}$ & 252.36 & 41.05 & 50.45 & 57.493 & 7.10 & 2.76 \\
\hline $\mathbf{T}_{2} \mathbf{V}_{2}$ & 250.63 & 40.00 & 48.56 & 56.537 & 6.92 & 2.71 \\
\hline$T_{3} V_{2}$ & 241.75 & 39.01 & 47.90 & 55.157 & 6.69 & 2.64 \\
\hline $\mathbf{T}_{4} \mathbf{V}_{2}$ & 231.99 & 35.02 & 45.83 & 54.837 & 6.54 & 2.55 \\
\hline $\mathbf{T}_{5} \mathbf{V}_{2}$ & 245.57 & 36.02 & 45.08 & 53.697 & 6.43 & 2.51 \\
\hline$T_{6} V_{2}$ & 236.43 & 39.05 & 43.43 & 52.757 & 6.30 & 2.43 \\
\hline $\mathbf{T}_{7} \mathbf{V}_{2}$ & 229.44 & 36.04 & 43.02 & 51.883 & 6.03 & 2.37 \\
\hline$T_{8} V_{2}$ & 223.52 & 36.06 & 40.72 & 51.09 & 5.90 & 2.31 \\
\hline $\mathbf{T}_{1} \mathbf{V}_{3}$ & 261.25 & 42.01 & 47.84 & 55.19 & 6.95 & 2.42 \\
\hline $\mathbf{T}_{2} \mathbf{V}_{3}$ & 257.89 & 41.04 & 47.10 & 53.743 & 6.83 & 2.36 \\
\hline $\mathbf{T}_{\mathbf{3}} \mathbf{V}_{\mathbf{3}}$ & 253.22 & 39.05 & 46.19 & 53.11 & 6.58 & 2.29 \\
\hline$T_{4} V_{3}$ & 243.23 & 38.00 & 44.57 & 51.967 & 6.44 & 2.21 \\
\hline $\mathbf{T}_{5} \mathbf{V}_{3}$ & 248.54 & 39.01 & 43.78 & 50.833 & 6.32 & 2.16 \\
\hline$T_{6} V_{3}$ & 246.46 & 39.02 & 42.98 & 50.183 & 6.19 & 2.02 \\
\hline $\mathbf{T}_{7} \mathbf{V}_{\mathbf{3}}$ & 239.49 & 37.02 & 41.77 & 49.367 & 5.93 & 1.93 \\
\hline$T_{8} V_{3}$ & 235.49 & 36.05 & 40.16 & 48.647 & 5.78 & 1.84 \\
\hline CD & 4.79 & 0.85 & 0.67 & 0.99 & 0.12 & 0.02 \\
\hline $\operatorname{S.Em}( \pm)$ & 1.67 & 0.30 & 0.23 & 0.35 & 0.04 & 0.01 \\
\hline
\end{tabular}

Note: $\mathrm{T}_{1}=$ Mustard oil cake $(50 \%)+$ Urea $(50 \%), \mathrm{T}_{2}=$ Cow dung manure $(50 \%)+$ Urea $(50 \%), \mathrm{T}_{3}=$ Poultry manure $(50 \%)+$ Urea $(50 \%), \mathrm{T}_{4}=$ Pond silt $(50 \%)+$ Urea $(50 \%), \mathrm{T}_{5}=$ Mustard oil cake $(100 \%), \mathrm{T}_{6}=$ Cow dung manure $(100 \%)$, $\mathrm{T}_{7}=$ Poultry manure $(100 \%), \mathrm{T}_{8}=$ Pond silt $(100 \%)$, Simurali Deshi $\left(\mathrm{V}_{1}\right)$, Simurali Bhabna $\left(\mathrm{V}_{2}\right)$ and Halisahar Sanchi $\left(\mathrm{V}_{3}\right)$ 
Table.4 Effect of different nitrogen sources on economy of three cultivars of betelvine

\begin{tabular}{|c|c|c|c|c|c|}
\hline Treatments & Cultivars & $\begin{array}{l}\text { Gross Return/ } \\
\text { ha/ year (Rs) }\end{array}$ & $\begin{array}{l}\text { Total } \\
\text { Cost/ha/ } \\
\text { year }\end{array}$ & $\begin{array}{c}\text { Net } \\
\text { Return/ha/ } \\
\text { year (Rs) }\end{array}$ & $\begin{array}{c}\text { Benefit: } \\
\text { Cost }\end{array}$ \\
\hline \multirow[t]{3}{*}{$\mathbf{T}_{1}$} & $V_{1}$ & 210721 & 65847 & 144875 & 2.20 \\
\hline & $V_{2}$ & 197479 & 65847 & 131632 & 2.00 \\
\hline & $\mathbf{V}_{3}$ & 199718 & 65847 & 133871 & 2.03 \\
\hline \multirow[t]{3}{*}{$\mathbf{T}_{2}$} & $V_{1}$ & 180009 & 64433 & 115576 & 1.79 \\
\hline & $\mathbf{V}_{2}$ & 164126 & 64433 & 99692 & 1.55 \\
\hline & $\mathbf{V}_{3}$ & 165973 & 64433 & 101539 & 1.58 \\
\hline \multirow[t]{3}{*}{$\mathbf{T}_{3}$} & $V_{1}$ & 148587 & 62813 & 85774 & 1.37 \\
\hline & $V_{2}$ & 136999 & 62813 & 74186 & 1.18 \\
\hline & $V_{3}$ & 147690 & 62813 & 84877 & 1.35 \\
\hline \multirow[t]{3}{*}{$\mathbf{T}_{4}$} & $V_{1}$ & 142879 & 63087 & 79792 & 1.26 \\
\hline & $V_{2}$ & 131845 & 63087 & 68758 & 1.09 \\
\hline & $\mathbf{V}_{3}$ & 132183 & 63087 & 69096 & 1.10 \\
\hline \multirow[t]{3}{*}{$\mathbf{T}_{5}$} & $V_{1}$ & 139671 & 68920 & 70751 & 1.03 \\
\hline & $V_{2}$ & 131990 & 68920 & 63070 & 0.92 \\
\hline & $\mathbf{V}_{3}$ & 139367 & 68920 & 70447 & 1.02 \\
\hline \multirow[t]{3}{*}{$\mathbf{T}_{6}$} & $V_{1}$ & 124744 & 66253 & 58490 & 0.88 \\
\hline & $V_{2}$ & 111965 & 66253 & 45712 & 0.69 \\
\hline & $\mathbf{V}_{3}$ & 114556 & 66253 & 48302 & 0.73 \\
\hline \multirow[t]{3}{*}{$\mathbf{T}_{7}$} & $V_{1}$ & 112838 & 63347 & 49491 & 0.78 \\
\hline & $V_{2}$ & 102895 & 63347 & 39548 & 0.62 \\
\hline & $\mathbf{V}_{3}$ & 111050 & 63347 & 47703 & 0.75 \\
\hline \multirow[t]{3}{*}{$\mathbf{T}_{8}$} & $V_{1}$ & 105785 & 63440 & 42345 & 0.67 \\
\hline & $V_{2}$ & 102390 & 63440 & 38950 & 0.61 \\
\hline & $\mathbf{V}_{3}$ & 104859 & 63440 & 41419 & 0.65 \\
\hline
\end{tabular}

Note: $\mathrm{T}_{1}=$ Mustard oil cake $(50 \%)+$ Urea $(50 \%), \mathrm{T}_{2}=$ Cow dung manure $(50 \%)+$ Urea $(50 \%), \mathrm{T}_{3}=$ Poultry manure $(50 \%)+$ Urea $(50 \%), \mathrm{T}_{4}=$ Pond silt $(50 \%)+$ Urea $(50 \%), \mathrm{T}_{5}=$ Mustard oil cake $(100 \%), \mathrm{T}_{6}=$ Cow dung manure $(100 \%), \mathrm{T}_{7}=$ Poultry manure $(100 \%), \mathrm{T}_{8}=$ Pond silt $(100 \%)$, Simurali Deshi $\left(\mathrm{V}_{1}\right)$, Simurali Bhabna $\left(\mathrm{V}_{2}\right)$ and Halisahar Sanchi $\left(\mathrm{V}_{3}\right)$

\section{Qualitative characters}

\section{Fresh and dry weight of 100 leaves}

Data presented in Table-3 clearly indicated significant variation with respect to fresh and dry weight of 100 leaves. Combination of mustard oil cake (50\%) and urea (50\%) produced maximum fresh weight in Halisahar Sanchi $\left(\mathrm{V}_{3}\right)(261.25 \mathrm{~g})$ followed by Simurali Bhabna $\left(\mathrm{V}_{2}\right)$ (252.36 g) and Simurali Deshi $\left(\mathrm{V}_{1}\right)(247.91 \mathrm{~g})$. Minimum fresh weight of 100 leaves was observed when nitrogen was applied in the form of pond silt (100\%) and the fresh weights were $220.60 \mathrm{~g}$ in Simurali Deshi $\left(\mathrm{V}_{1}\right), 223.52 \mathrm{~g}$ in Simurali Bhabna $\left(\mathrm{V}_{2}\right)$ and $235.49 \mathrm{~g}$ in Halisahar Sanchi $\left(\mathrm{V}_{3}\right)$. Among the three cultivars, maximum fresh weight was found in Halisahar Sanchi $\left(\mathrm{V}_{3}\right)$ (248.20 g).

Mustard oil cake $(50 \%)+$ urea $(50 \%)$ is one of the treatment combination which produced maximum dry weight of 100 leaves in Halisahar Sanchi $\left(\mathrm{V}_{3}\right)$ (42.01 g), Simurali Bhabna $\left(\mathrm{V}_{2}\right)(41.05 \mathrm{~g})$ and Simurali Deshi 
$\left(\mathrm{V}_{1}\right)$ (40.04 g) while minimum dry weight was produced where pond silt was used as $100 \%$ nitrogen source. Among the cultivars, Halisahar Sanchi $\left(\mathrm{V}_{3}\right)$ produced maximum dry weight of 100 leaves $(38.90 \mathrm{~g}$ ). Among all of the treatment combinations, maximum dry weight $(41.03 \mathrm{~g})$ was found from mustard oil cake $(50 \%)$ and urea $(50 \%)$.

\section{Chlorophyll content of leaves (SPAD value)}

Combination of mustard oil cake $(50 \%)$ and urea $(50 \%)$ produced maximum chlorophyll content of the leaves in Simurali Deshi $\left(\mathrm{V}_{1}\right)$ (52.30) followed by Simurali Bhabna $\left(V_{2}\right)$ (50.45) and Halisahar Sanchi $\left(\mathrm{V}_{3}\right)$ (47.84). Minimum chlorophyll content of leaf was observed when nitrogen was applied in the form of pond silt $(100 \%)$ and the results were as 41.76 in Simurali Deshi $\left(\mathrm{V}_{1}\right), 40.72$ in Simurali Bhabna $\left(\mathrm{V}_{2}\right)$ and 40.16 in Halisahar Sanchi $\left(\mathrm{V}_{3}\right)$. Among the three cultivars, maximum chlorophyll content was found in Simurali Deshi $\left(\mathrm{V}_{1}\right)$ (46.72). Among all of the treatment combinations, mustard oil cake $(50 \%)$ and urea $(50 \%)$ showed the highest result (50.19).

The application of organic manures and inorganic fertilizers in combination lowered the $\mathrm{C}: \mathrm{N}$ ratio thereby favouring microbial decomposition which in turn contributed to the higher uptake of nitrogen along with other nutrients, leading to higher level of photosynthetic pigments. (Arulmozahiyan et al., 1998) ${ }^{[3]}$.

\section{Ascorbic acid content of leaves (mg /100g)}

Combination of mustard oil cake $(50 \%)$ and urea $(50 \%)$ produced highest Ascorbic acid content of leaves in Simurali Deshi $\left(\mathrm{V}_{1}\right)$ (3.07 $\mathrm{mg} / 100 \mathrm{~g})$ followed by Simurali Bhabna $\left(\mathrm{V}_{2}\right)$ $(2.76 \mathrm{mg} / 100 \mathrm{~g})$ and Halisahar Sanchi $\left(\mathrm{V}_{3}\right)$ (2.42 mg/100g). Lowest Ascorbic acid content was found in Halisahar Sanchi $\left(\mathrm{V}_{3}\right)$
(1.84 mg /100g) from $\mathrm{T}_{8}$ i.e. pond silt (100\%). All-over among the cultivars Simurali Deshi $\left(\mathrm{V}_{1}\right)$ produced highest $(2.82 \mathrm{mg} / 100 \mathrm{~g})$ Ascorbic acid content followed by Simurali Bhabna $\left(\mathrm{V}_{2}\right)(2.54 \mathrm{mg} / 100 \mathrm{~g})$ and Halisahar Sanchi $\quad\left(\mathrm{V}_{3}\right) \quad(2.16 \mathrm{mg} / 100 \mathrm{~g})$. Overall performance was best $(2.75 \mathrm{mg} / 100 \mathrm{~g})$ when nitrogen was supplied as a combination of mustard oil cake (50\%) and urea (50\%). Guha $(2006)^{[11]}$ reported that ascorbic acid content in fresh betel leaves varied from $0.002 \%-$ $0.01 \%$.

\section{Essential oil content of leaves (mg/100g)}

Mustard oil cake 50\% + Urea 50\% $\left(\mathrm{T}_{1}\right)$ was found to be the best in case of essential oil production and results were as $65.50 \mathrm{mg} / 100 \mathrm{~g}$ in Simurali Deshi $\left(\mathrm{V}_{1}\right)$ followed by 57.49 $\mathrm{mg} / 100 \mathrm{~g}$ in Simurali Bhabna $\left(\mathrm{V}_{2}\right)$ and 55.74 $\mathrm{mg} / 100 \mathrm{~g}$ in Halisahar Sanchi $\left(\mathrm{V}_{3}\right)$. The lowest oil content was obtained with application of pond silt as $100 \%$ nitrogen source $\left(\mathrm{T}_{8}\right)$. The overall result showed that the highest essential oil content $(59.39 \mathrm{mg} / 100 \mathrm{~g})$ was obtained with application of mustard oil cake $(50 \%)$ and urea $(50 \%)$. Among the cultivars, Simurali Deshi $\left(\mathrm{V}_{1}\right)$ produced highest essential oil content (60.68 mg/100g). Essential oil is a secondary plant metabolite synthesized from the products of photosynthesis. Restricted availability of photosynthates adversely affects crop growth and essential oil synthesis and accumulation (Kumar et al., 2014) ${ }^{[20]}$.

\section{B Carotene content of leaves (mg/100g)}

Mustard oil cake 50\% + Urea 50\% ( $\left.\mathrm{T}_{1}\right)$ was found to be the best, resulting $7.28 \mathrm{mg} / 100 \mathrm{~g}$ in Simurali Deshi $\left(\mathrm{V}_{1}\right), 7.10 \mathrm{mg} / 100 \mathrm{~g}$ in Simurali Bhabna $\left(\mathrm{V}_{2}\right)$ and $6.95 \mathrm{mg} / 100 \mathrm{~g}$ in Halisahar Sanchi $\left(V_{3}\right)$ in production of $B$ Carotene content of leaves. The lowest result was obtained with application of pond silt as $100 \%$ nitrogen source $\left(\mathrm{T}_{8}\right)$. The overall result 
showed that the maximum $B$ Carotene content $(7.11 \mathrm{mg} / 100 \mathrm{~g})$ was obtained with application of mustard oil cake $(50 \%)$ and urea $(50 \%)$ and minimum B Carotene content $(5.94 \mathrm{mg} / 100 \mathrm{~g}$ ) was obtained when $100 \%$ nitrogen was applied from pond silt $\left(\mathrm{T}_{8}\right)$. Among the cultivars, Simurali Deshi $\left(\mathrm{V}_{1}\right)$ produced maximum $B$ Carotene content $(6.75 \mathrm{mg} / 100 \mathrm{~g})$.

\section{Economy of the crop}

Data presented in Table-4 clearly indicated that the benefit and cost ratio varied with the nitrogen sources (treatments) and cultivars in betel vine. Among the sources of nitrogen Mustard oil cake $50 \%$ + Urea $50 \%\left(\mathrm{~T}_{1}\right)$ showed highest benefit and cost ratio in all the three cultivars with the values of 2.20 in Simurali Deshi $\left(\mathrm{V}_{1}\right), 2.00$ IN Simurali Bhabna $\left(\mathrm{V}_{2}\right)$ and 2.03 in Halisahar Sanchi $\left(\mathrm{V}_{3}\right)$ followed by cowdung manure $50 \%+$ Urea $50 \%\left(\mathrm{~T}_{2}\right)$ resulting $1.79,1.55$ and 1.58 in Simurali Deshi $\left(\mathrm{V}_{1}\right)$, Simurali Bhabna $\left(\mathrm{V}_{2}\right)$ and Halisahar Sanchi $\left(\mathrm{V}_{3}\right)$ respectively. The lowest benefit and cost ratio in all the three cultivars where 0.61 in Simurali Bhabna $\left(\mathrm{V}_{2}\right)$, 0.65 in Halisahar Sanchi $\left(\mathrm{V}_{3}\right)$ and 0.67 in Simurali Deshi $\left(\mathrm{V}_{1}\right)$, when $100 \%$ nitrogen was applied from Pond silt. In benefit and cost ratio Simurali Deshi $\left(\mathrm{V}_{1}\right)$ performed the best followed by Halisahar Sanchi $\left(\mathrm{V}_{3}\right)$ and Simurali Bhabna $\left(\mathrm{V}_{2}\right)$. When $100 \%$ nitrogen was applied from mustard oil cake showed a benefit and cost ratio of 2.20 in Simurali Deshi $\left(\mathrm{V}_{1}\right), 0.92$ in Simurali Bhabna $\left(\mathrm{V}_{2}\right)$ and 1.02 in Halisahar Sanchi $\left(\mathrm{V}_{3}\right)$.

\section{References}

1. APEDA agriXchange: the changing face of agri-business.

https://agriexchange.apeda.gov.in/product_p rofile/exp_f_india.aspx? categorycode $=0207$ \#

2. Arnon, D. I. (1949). Copper enzymes in isolated chloroplasts. Polyphenoloxidase in Beta vulgaris. Plant physiology, 24(1), 1.
3. Arulmozhiyan, R., Chitra, R., Prabhakar, K., Jalaluddin, S.M. and Packiaraj, D. (2005). SGM. BV.2 - A new promising betel vine variety. Madras Agric. J. 92(2): 498-503.

4. Boardman, N. K., and Anderson, J. M. (1967). Fractionation of the photochemical systems of photosynthesis. II. Cytochrome and carotenoid contents of particles isolated from spinach chloroplasts. Biochimica et Biophysica Acta (BBA)-Bioenergetics, 143(1), 187-203.

5. Chang, M. C., Uang, B. J., Wu, H. L., Lee, J. J., Hahn, L. J. and Jeng, J. H. (2002). Inducing the cell cycle arrest and apoptosis of oral KB carcinoma cells by hydroxychavicol: roles of glutathione and reactive oxygen species. British journal of pharmacology, 135(3), 619-630.

6. Clevenger, J. F. (1928). Apparatus for the determination of volatile oil. Journal of the American Pharmaceutical Association, 17(4), 345-349.

7. Das, B. K. and Mallick, S. K. (2010). "Screening of Betel vine Cultivars for Resistance to Betel vine Whitefly, Singhiella pallida (Singh) (Hemiptera: Aleyrodidae) and New Host Plant Records". Pest Management in Horticultural Ecosystems. 16: 87-94.

8. Dey, M., Pariari, A., Sharangi, A.B. and Chatterjee, R. (2003). Response of different nitrogen sources on growth and yield of betelvine (Piper betle L.). South Indian Horticulture, 51(1/6): 244-248.

9. Ghosh, R., Nadiminty, N., Fitzpatrick, J. E., Alworth, W. L., Slaga, T. J. and Kumar, A. P. (2005). Eugenol causes melanoma growth suppression through inhibition of E2F1 transcriptional activity. Journal of Biological Chemistry, 280(7), 5812-5819.

10. Gomez, K. A. and Gomez, A. A. (1984). Statistical procedures for agricultural research. John Wiley \& Sons.

11. Guha, P. (2006). Betel leaf: the neglected green gold of India. Journal of Human Ecology, 19(2), 87-93.

12. Gundala, S. R., Yang, C., Mukkavilli, R., Paranjpe, R., Brahmbhatt, M., Pannu, V. and Aneja, R. (2014). Hydroxychavicol, a betel leaf component, inhibits prostate cancer through ROS-driven DNA damage and 
apoptosis. Toxicology and applied pharmacology, 280(1), 86-96.

13. Imam, M.N., Pariari, A. and Sahu, P.K. (2012). Effect of integrated nitrogen application on growth and yield of betelvine (Piper betle L.). Journal of Crop and Weed, 8(1): 141-144.

14. Jaganathan, S. K., Mazumdar, A., Mondhe, D. and Mandal, M. (2011). Apoptotic effect of eugenol in human colon cancer cell lines. Cell biology international, 35(6), 607615.

15. Jane, N. S., Deshmukh, A. P., \& Joshi, M. S. (2014). Review of study of different diseases on betelvine plant and control measure. International Journal of Application or Innovation in Engineering \& Management, 3(3), 560-563.

16. Jeng, J. H., Wang, Y. J., Chang, W. H., Wu, H. L., Li, C. H., Uang, B. J. and Chang, M. C. (2004). Reactive oxygen species are crucial for hydroxychavicol toxicity toward KB epithelial cells. Cellular and Molecular Life Sciences CMLS, 61(1), 83-96.

17. Kaleeswari, V. and Sridhar, T. A (2013). Study on Betelvine cultivation and market crisis in Karur district. International Journal of Applied Research and Studies; 3(10): 1-3.

18. Kassi, E., Papoutsi, Z., Pratsinis, H., Aligiannis, N., Manoussakis, M. and Moutsatsou, P. (2007). Ursolic acid, a naturally occurring triterpenoid, demonstrates anticancer activity on human prostate cancer cells. Journal of cancer research and clinical oncology, 133(7), 493500.

19. Khatua, D. C., Mondal, B. and Bhattachayya, R. 2013. A selective medium for Xanthomonas axonopodis pv. Betlicola, bacterial pathogen of betelvine. African Journal of Agricultural Research. 8(49): 6388-6393.

20. Kumar, V., Swamy, K. N., Vardhini, B. V. and Rao, S. S. R. (2014). Effect of light curtailment on growth, biochemical response and essential oil content of rose scented geranium. International Journal of Multidisciplinary and Current Research, 2, 322-326.

21. Maiti, S., Kadam, A.S., Sengupta, K., Punekar, L.K., Das, J.N., Saikia, L., Biswas,
S.R. and Reddy, K.M. (1995). Effect of sources and levels of nitrogen on growth and yield of betelvine (Piper betle L.). Journal of Plantation Crops, 23 (2): 122- 125.

22. Manikandan, P., Murugan, R. S., Priyadarsini, R. V., Vinothini, G. and Nagini, S. (2010). Eugenol induces apoptosis and inhibits invasion and angiogenesis in a rat model of gastric carcinogenesis induced by MNNG. Life sciences, 86(25-26), 936941.

23. Paranjpe, R., Gundala, S. R., Lakshminarayana, N., Sagwal, A., Asif, G., Pandey, A., \& Aneja, R. (2013). Piper betel leaf extract: anticancer benefits and bioguided fractionation to identify active principles for prostate cancer management. Carcinogenesis, 34(7), 1558-1566.

24. Pisano, M., Pagnan, G., Loi, M., Mura, M. E., Tilocca, M. G., Palmieri, G. and Rozzo, C. (2007). Antiproliferative and proapoptotic activity of eugenol-related biphenyls on malignant melanoma cells. Molecular cancer, 6(1), 8 .

25. Prasad, B. and Prasad, S. (2003) Pattern of employment in betelvine cultivation. Journal of Applied Biology, 13(1/2):125-129.

26. Punuri, J. B., Sharma, P., Sibyala, S., Tamuli, R., \& Bora, U. (2012). Piper betlemediated green synthesis of biocompatible gold nanoparticles. International Nano Letters, 2(1), 18.

27. Rai, M. P., Thilakchand, K. R., Palatty, P. L., Rao, P., Rao, S., Bhat, H. P. and Baliga, M. S. (2011). Piper betel Linn (betel vine), the maligned Southeast Asian medicinal plant possesses cancer preventive effects: Time to reconsider the wronged opinion. Asian Pac J Cancer Prev, 12(9), 2149-2156.

28. Rakshit, S., Mandal, L., Pal, B. C., Bagchi, J., Biswas, N., Chaudhuri, J. and Mukherjee, T. (2010). Involvement of ROS in chlorogenic acid-induced apoptosis of BcrAbl+ CML cells. Biochemical pharmacology, 80(11), 1662-1675.

29. Ranganna, S. (2000). Handbook of canning and aseptic packaging. Tata McGraw-Hill Publishing Company.

30. Ray, D.P. (2008). Keynote Address, National Seminar on Piperaceae, 21-22 
November 2008, IISR, Calicut. p. 26.

31. Sahoo, M. and Sahoo D.R. (2017). Betel Leaf Cultivation in Odisha: Problems and Prospects. Asian Review of Social Sciences;7(1):10-15.

32. Saikia, L., Bhuyan, C.K. and Dutta, P.K. (1995). Study on growth, yield and keeping quality of betelvine (Piper betle L.) $c v$. Local Bangla as influenced by source and level of nitrogenous fertilizers. Indian cocoa, Arecanut and Spices Journal, 19(2): 46-50.

33. Satyavati, G. V., Raina, M. K. and Sharma, M. (1987). Medicinal plants of India. Indian Council of Medical Research.

34. Sengupta, S.K., Chaurasia, R.K. and Bhatt, J. (2004). Influence of organic and inorganic nutrition on the productivity of betelvine crop and storage life of betel leaves (Piper betel L.). South Indian Horticulture, 52(1/6): 263-269.

35. Shao, J. W., Dai, Y. C., Xue, J. P., Wang, J. C., Lin, F. P. and Guo, Y. H. (2011). In vitro and in vivo anticancer activity evaluation of ursolic acid derivatives. European journal of medicinal chemistry, 46(7), 2652-2661.

36. Shukla, U. N., Khakare, M. S., Srivastava, V. K., Kumar, R., Singh, S., Kumar, V. and Kumar, K. (2013). Effect of spacings and fertility levels on growth, yield and quality of cotton (Gossypium hirsutum L.) hybrids under rainfed condition of vidarbha. The Bioscan. 8(2): 561-567.

37. Thomas, U. C., Chandini, S., \& Thomas, A.
(2013). Integrated Nutrient Management Studies in betelvine (Piper betel L.). International Journal of Applied Research and Studies, 2(7).

38. Warrier, P. K., Nambiar, V. P. K., and Ramankutty, C. (1995). Indian medical plants.

39. Yadav, R. D. (2005). Integrated nutrient management in coriander (Coriandrum sativum L.) under loamy sand soils. Ph.D. Thesis, Rajasthan Agricultural University, Campus-Bikaner.

40. Yamai, H., Sawada, N., Yoshida, T., Seike, J., Takizawa, H., Kenzaki, K. and Tangoku, A. (2009). Triterpenes augment the inhibitory effects of anticancer drugs on growth of human esophageal carcinoma cells in vitro and suppress experimental metastasis in vivo. International journal of cancer, 125(4), 952-960.

41. Yu, Y. X., Gu, Z. L., Yin, J. L., Chou, W. H., Kwok, C. Y., Qin, Z. H. and Liang, Z. Q. (2010). Ursolic acid induces human hepatoma cell line SMMC-7721 apoptosis via p53-dependent pathway. Chinese medical journal, 123(14), 1915-1923.

42. Yuan- long, W., Gui-hu, L. and Guangdeng (2012). Effects of different shaded conditions and water depths on the growth and reproductive strategy of Vallisneria spinulosa. Pakistan Journal of Botany; 44: 911-918.

\section{How to cite this article:}

Aloke Bar, Anupam Pariari Pooja Biswas, Milan Karmakar and Gobinda Saha. 2020. Comparative Studies on Leaf Yield, Quality and Economy of Three Cultivars of Betelvine (Piper betle L.) as Influenced by Integrated Nitrogen Management. Int.J.Curr.Microbiol.App.Sci. 9(11): 3137-3147. doi: https://doi.org/10.20546/ijcmas.2020.911.378 